Document downloaded from:

http://hdl.handle.net/10251/43338

This paper must be cited as:

Mukherjee, S.; Silla Jiménez, F.; Bannon, P.; Emer, J.; Lang, S.; Webb, D. (2002). A comparative study of arbitration algorithms for the Alpha 21364 pipelined router. ACM SIGPLAN Notices. 37(10):223-234. doi:10.1145/605432.605421.

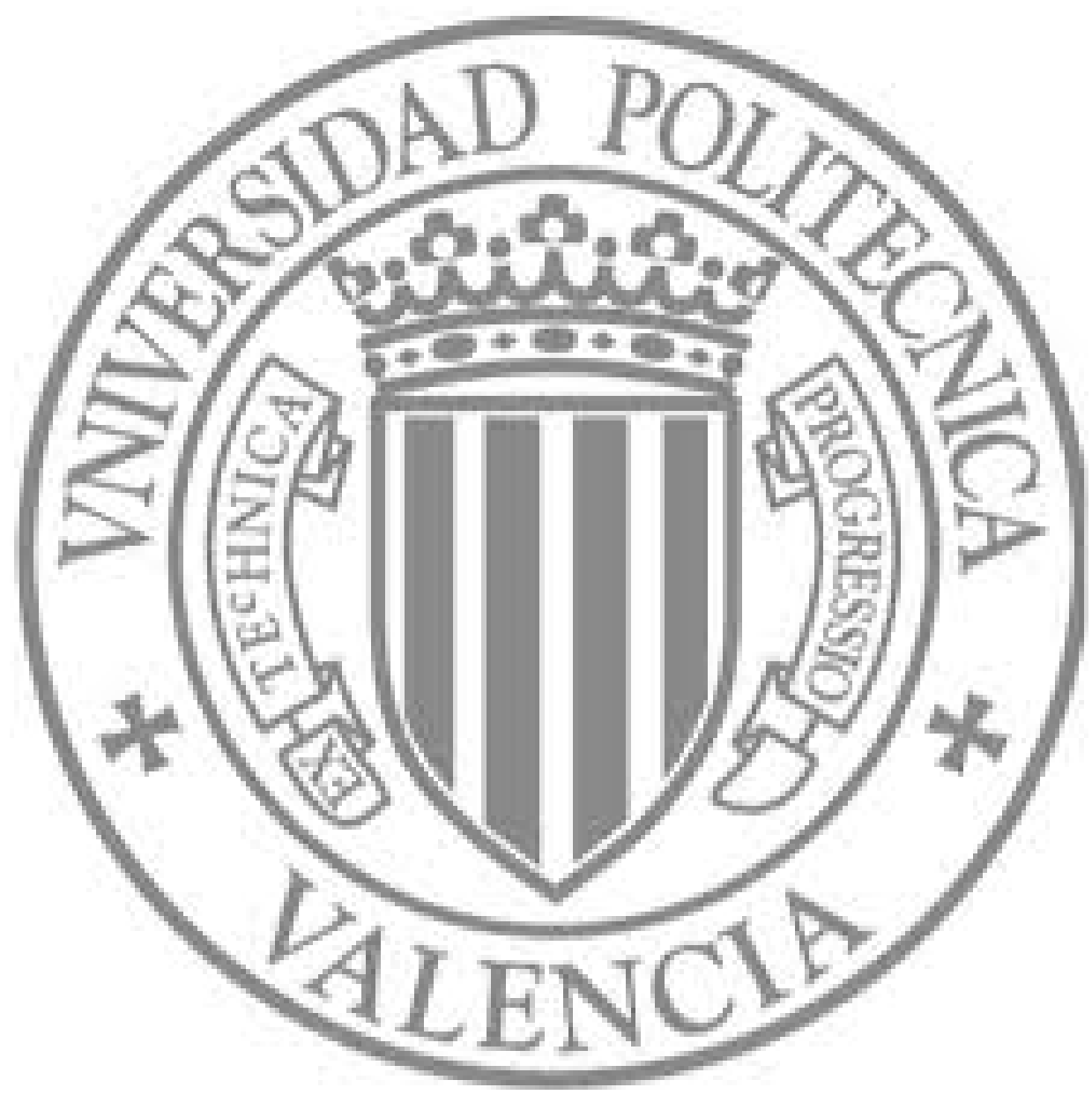

The final publication is available at

http://dx.doi.org/10.1145/605432.605421

Copyright ACM 


\title{
A Comparative Study of Arbitration Algorithms for the Alpha 21364 Pipelined Router
}

\author{
Shubhendu S. Mukherjee*, Federico Silla ${ }^{\$}$, Peter Bannon!, Joel Emer, Steve Lang ${ }^{\star}$, and David Webb! \\ *Intel Corporation \\ 334 South Street \\ Shrewsbury, MA 01545 \\ \{shubu.mukherjee,joel.emer,steve.lang\}@intel.com \\ ${ }^{\$}$ Department of Computer Engineering \\ Universidad Politecnica de Valencia \\ Camino de Vera s/n, 46022 Valencia, Spain \\ fsilla@disca.upv.es \\ 'Hewlett-Packard \\ 334 South Street \\ Shrewsbury, MA 01545 \\ \{peter.bannon,david.webb\}@hp.com
}

\begin{abstract}
Interconnection networks usually consist of a fabric of interconnected routers, which receive packets arriving at their input ports and forward them to appropriate output ports. Unfortunately, network packets moving through these routers are often delayed due to conflicting demand for resources, such as output ports or buffer space. Hence, routers typically employ arbiters that resolve conflicting resource demands to maximize the number of matches between packets waiting at input ports and free output ports. Efficient design and implementation of the algorithm running on these arbiters is critical to maximize network performance.

This paper proposes a new arbitration algorithm called SPAA (Simple Pipelined Arbitration Algorithm), which is implemented in the Alpha 21364 processor's on-chip router pipeline. Simulation results show that SPAA significantly outperforms two earlier well-known arbitration algorithms: PIM (Parallel Iterative Matching) and WFA (Wave-Front Arbiter) implemented in the SGI Spider switch. SPAA outperforms PIM and WFA because SPAA exhibits matching capabilities similar to PIM and WFA under realistic conditions when many output ports are busy, incurs fewer clock cycles to perform the arbitration, and can be pipelined effectively. Additionally, we propose a new prioritization policy called the Rotary Rule, which prevents the network's adverse performance degradation from saturation at high network loads by prioritizing packets already in the network over new packets generated by caches or memory.
\end{abstract}

\section{INTRODUCTION}

Cache-coherent, shared-memory multiprocessors with 16 or more processors have become common server machines. In 2001 such machines generated a total revenue of $\$ 9$ billion, which is roughly $16 \%$ of the world-wide server revenue [6]. This market segment's revenue tripled in the last four years making it the fastest growing segment of the entire server market. Major vendors, such as IBM [8][37], Hewlett-Packard [19][17][26], SGI [33], and Sun Microsystems [6] offer such shared-memory multiprocessors, which scale up to anywhere between 24 and 512 processors.

High performance interconnection networks are critical to the success of large-scale shared-memory multiprocessors. Such

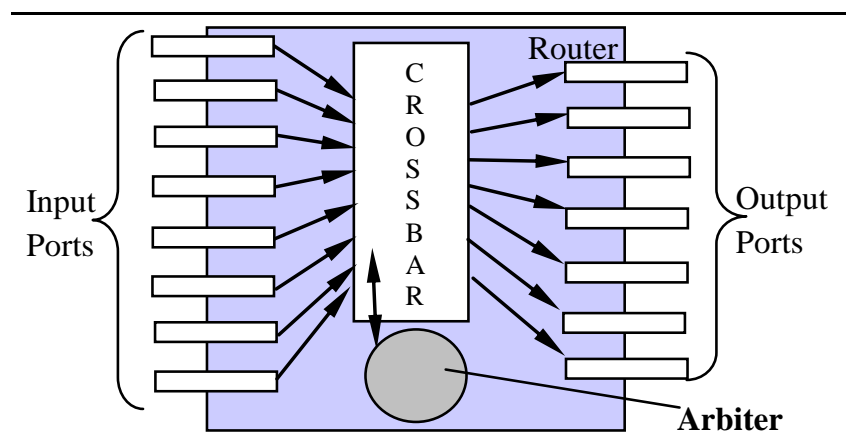

Figure 1. A router with 8 input ports and 7 output ports, like the Alpha 21364 router. The arbiter controls how and which packets are forwarded from the input to the output ports.

networks allow a large number of processors and memory modules to communicate with one another using a cache coherence protocol. In such systems, a processor's cache miss to a remote memory module (or another processor's cache) and consequent miss response are encapsulated in network packets and delivered to the appropriate processors or memories. The performance of many parallel applications, such as database servers [29], depends on how rapidly and how many of these miss requests and responses can be processed by the system. Consequently, it is extremely critical for networks to deliver packets with low latency and high bandwidth.

An interconnection network usually consists of a fabric of small interconnected routers, which receive packets arriving at their input ports and forward them to appropriate output ports. Unfortunately, packets moving through such routers are often delayed due to conflicting demand for resources, such as output ports or buffer space. Hence, routers include arbiters to resolve conflicting resource demands (Figure 1). The presence of input buffers in a router usually divides up the arbitration process into two steps: first an input port picks one or more packets from those waiting in its buffers, and then an output port picks a packet among the packets nominated to it by one or more input ports. By definition only one packet can be delivered through an output port.

This paper examines several arbitration algorithm choices for the on-chip router in the Alpha 21364 processor [3], which runs at $1.2 \mathrm{GHz}$ and uses 152 million transistors to integrate on the same chip an aggressive dynamically-scheduled processor, 1.75 megabytes of second-level cache, two Rambus Direct RDRam ${ }^{\mathrm{TM}}$ memory controllers, and an interconnection network router. Efficient design and implementation of these arbiters is critical to maximize network throughput, as illustrated by Figure 2. Typically, arbitration algorithms try to maximize the number of matches between input and output ports to provide high local routing throughput. A locally maximal match in a router does not necessarily guarantee globally optimal network 


\begin{tabular}{|l|l|l|l|}
\hline Input Port 0 & 3 & 2 & 1 \\
\hline Input Port 1 & 3 & 2 & 1 \\
\hline Input Port 2 & 3 & 2 & 1 \\
\hline Input Port 3 & 3 & 2 & 1 \\
\hline Input Port 4 & 3 & 6 & 1 \\
\hline Input Port 5 & 3 & 2 & 0 \\
\hline Input Port 6 & 3 & 2 & 4 \\
\hline Input Port 7 & 3 & 2 & 5 \\
\hline
\end{tabular}

Figure 2. An illustration of the challenges of an arbitration algorithm for the router in Figure 1. The first column lists the input ports. Column $2-4$ list the output port destinations of the packets waiting at that input port. Column 2 contains the oldest packets to arrive at the specific input port, while column 4 lists the corresponding youngest packets. An arbitration algorithm (lets call it $O P F$ ) that picks the oldest packet from each input port will be sub-optimal because output port 3 can deliver only one packet. Thus, OPF will result in arbitration collisions at output port 3 . In contrast, an arbitration algorithm that chooses the shaded packets will have the maximum throughput at this router in the current arbitration cycle.

performance. Nevertheless, in our experience, a locally maximal match has the first order impact on overall network performance.

The high-frequency implementation of the Alpha 21364 router made the already difficult task of arbitration even harder. The entire 21364 chip, including the router, runs at $1.2 \mathrm{GHz}$. In contrast, earlier generations of such routers ran at much slower speeds. For example, the Cray T3E router runs at $75 \mathrm{MHz}$ [31], the SGI Spider runs at $100 \mathrm{MHz}$ [16], while IBM's third generation Vulcan switch runs at $125 \mathrm{MHz}$ [35].

For efficient implementation at $1.2 \mathrm{GHz}$, we had to pipeline the 21364 router. Unfortunately, in the 0.18 micron CMOS process that the 21364 was designed for, only up to 12-13 logic levels could be incorporated in the 0.83 nanoseconds cycle time. This forced us to pipeline the arbitration algorithm itself, unlike the SGI switch in which the algorithm was implemented within one 10-nanosecond clock cycle. Unfortunately, each additional cycle added to the 21364 router's arbitration pipeline degraded the network throughput by roughly $5 \%$ under heavy $\operatorname{load}^{1}$. Hence, any additional cycles incurred by a more complex arbitration algorithm must gain back the performance degradation from the added cycles in the pipeline.

This paper shows that SPAA (Simple Pipelined Arbitration Algorithm) - implemented in the 21364 router-significantly outperforms two well-known arbitration algorithms-Parallel Iterative Matching (PIM) [2] and Wavefront Arbitration (WFA) [36], which is implemented in the SGI Spider switch. For completeness, we also examine a maximal cardinality matching algorithm (MCM), which maximizes the number of matches between packets waiting at the input ports and free output ports.

The number of matches found by PIM and WFA between packets waiting at input ports and free output ports is close to that of MCM's, which makes both PIM and WFA very powerful arbitration algorithms. PIM iterates between the input and output ports to find a suitable match of packets, whereas WFA makes a pass through a matrix of input and output ports to find a suitable match.

The key to PIM and WFA's high matching capabilities lies in their high level of interaction between input and output ports.

\footnotetext{
${ }^{1}$ This measurement was done using SPAA, which is explained later.
}

When multiple input ports nominate packets to the same output port, naïve algorithms, such as OPF in Figure 2, can result in arbitration "collisions" and consequent poor performance. In contrast, both PIM and WFA's input and output port arbiters will interact to choose the appropriate match for the specific arbitration cycle. Unfortunately, such high level of interaction requires a higher number of cycles to implement them compared to what a simpler algorithm, such as SPAA, would need. Additionally, such interaction also makes it hard to pipeline these algorithms.

SPAA is a much simpler algorithm compared to PIM and WFA and is more like the OPF algorithm in Figure 2. In SPAA, each input port chooses a packet in every cycle to nominate to an output port. However, an input port arbiter's choice is independent of most of the other input port arbiters. Similarly, an output port arbiter chooses a packet from the packets nominated to it by the input port arbiters. But, an output port arbiter's decision is independent of the other output port arbiters' decisions. Thus, SPAA minimizes interactions between the input and output ports.

Clearly, because of its reduced interaction between input and output port arbiters, SPAA can result in arbitration collisions at the output port and, hence, fewer matches than what PIM or WFA would offer. Nevertheless, SPAA significantly outperforms both algorithms because of three reasons. First, with medium to heavy loads many output ports are busy and, hence, an arbitration algorithm need only find matches for a few free output ports. Thus, when our seven output ports are busy $50 \%$ of the time, SPAA's matching capabilities are similar to PIM and WFA's. The difference between PIM, WFA, and SPAA's matching capabilities is negligible when the output ports are busy $75 \%$ of the time.

Second, SPAA minimizes its interaction between input and output ports, which lowers its matching capabilities, but makes it simpler, so that it can be implemented in three cycles in the 21364 router. WFA would have incurred four cycles to implement. Similarly, one iteration of PIM takes four cycles to implement. Multiple iterations of PIM would have incurred significantly more cycles and would have obviously performed poorly in our environment. Hence, we use only one iteration of PIM - which we call PIM1 - in all our timing evaluations.

Third, SPAA can be pipelined effectively because it minimizes interactions between the input and output ports. PIM1 requires an extra step of interaction between the output and input ports, whereas WFA requires interaction between the output ports themselves. These features prevent both PIM1 and WFA from being pipelined effectively. In our implementation both PIM1 and WFA take four cycles, but can start input port arbitration every three cycles, whereas SPAA takes three cycles and can initiate input port arbitration every cycle.

Additionally, SPAA nominates a packet to only one output port, unlike PIM or WFA, which can nominate the same packet to multiple output ports. This has the added benefit that a packet can be speculatively read out from an input buffer as soon as it is scheduled for delivery by an input port arbiter (but before the output port arbitration is complete), much like the way direct-mapped caches allow processors to speculatively read out data before the address comparison completes [20].

Our simulation results show that SPAA significantly outperforms both PIM1 and WFA. We also demonstrate that SPAA will continue to deliver higher throughput compared to PIM1 


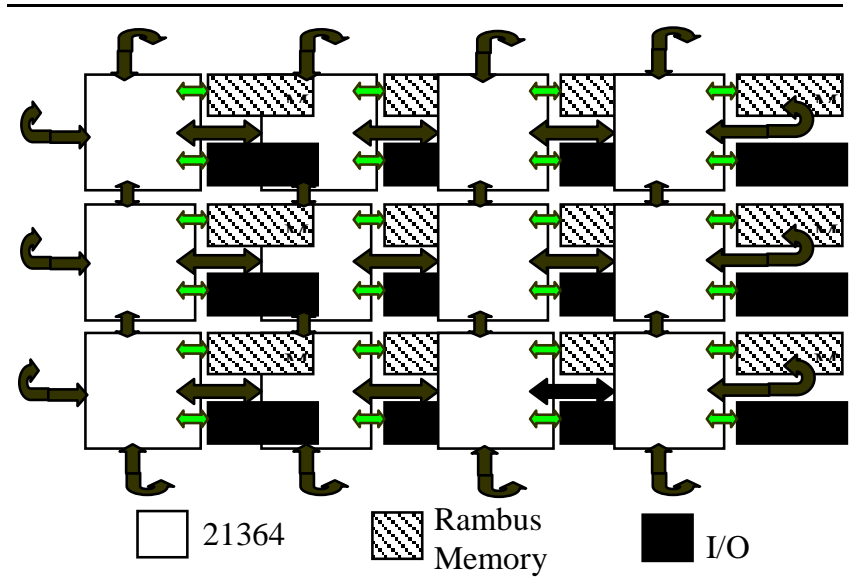

Figure 3. A 12-processor Alpha 21364 2D torus network.

and WFA, if the router were scaled to have twice the pipeline length, greater input load, or support bigger networks than the 21364 was designed for.

In addition to SPAA, we propose a new prioritization policy called the Rotary Rule, which provides a significant boost in network performance by preventing the network's adverse performance degradation from saturation at high network loads. The Rotary Rule prioritizes the movement of network packets already in the network over packets recently generated from the processor ports. We demonstrate the effectiveness of the Rotary Rule with WFA and SPAA. The Alpha 21364 router provides the Rotary Rule as an optional mode programmable at boot-time. We do not, however, expect most real applications running on a system composed of 21364 processors to create such heavy network load that would require us to turn on the Rotary Rule.

The rest of the paper is organized as follows. We first describe the 21364's base router architecture in Section 2. Section 3 discusses PIM, WFA, SPAA, and the Rotary Rule, as well as related work. Section 4 discusses our evaluation methodology and Section 5 describes our results. Section 6 summarizes the paper and presents our conclusions.

\section{THE ALPHA 21364 ON-CHIP ROUTER}

Mukherjee, et al. [26] discusses details of the 21364 network and router architectures. Here we summarize the salient features of the network (Section 2.1) and the router pipeline (Section 2.2) relevant to this paper.

\subsection{Network Architecture}

The Alpha 21364's on-chip router uses two million transistors to connect up to 128 processors in a two-dimensional torus network (Figure 3). Salient features of the network are:

- Packets. The network supports seven classes of coherence packets for the directory-based cache-coherence protocol. These are requests (three flits), forwards (three flits), block response (18 or 19 flits), non-block response (two or three flits), write I/O (19 flits), read I/O (three flits), and special (one flit, excluding no-ops). Each flit is 39 bits-32 bits for data and 7 bits for ECC. A 19 flit packet, such as a block response, can carry a 64-byte cache block (3 flits for header and 16 flits for the cache block). Thus, when an input or an output port is scheduled to deliver a packet, the port can be busy for two, three, 18, or 19 cycles. An output port is ready

\begin{tabular}{|c|c|c|c|c|c|c|c|c|c|}
\hline \multirow{2}{*}{ First Flit } & RT & $\mathrm{T}$ & DW & LA & $\mathrm{RE}$ & GA & \multirow{2}{*}{$X$} & \multirow{2}{*}{\multicolumn{2}{|c|}{ ECC }} \\
\hline & Nop & Nop & Nop & $\mathrm{WrQ}$ & $\mathrm{W}$ & RQ & & & \\
\hline \multicolumn{2}{|l|}{ Second Fl } & Nop & Nop & Nop & WrQ & W & RQ & $\mathrm{X}$ & ECC \\
\hline
\end{tabular}

(a)

First Flit

Second Flit

\begin{tabular}{|c|l|c|c|c|c|c|l|}
\hline ECC & DW & LA & RE & GA & \multirow{2}{*}{ X } & ECC & \\
\cline { 2 - 6 } & Nop & WrQ & W & RQ & & & \\
\hline & ECC & Nop & WrQ & W & RQ & X & ECC \\
\hline
\end{tabular}

(b)

Figure 4. Two of the nine logical router pipelines in the 21364. (a) shows the router pipeline for a local input port (cache or memory controller) to an interprocessor output port (b) shows the router pipeline from an interprocessor (north, south, east, or west) input port to an interprocessor output port. The first flit goes through two pipelines: the scheduling pipeline (upper pipeline) and data pipeline (lower pipeline). Second and subsequent flits follow the data pipeline. $R T=$ Router Table Lookup, Nop $=$ No operation, $T=$ Transport (wire delay), $D W=$ Decode and Write Entry Table, LA = Input Port Arbitration, $R E=$ Read Entry Table and Transport, GA = Output Port Arbitration, $W=$ Wait, $W r Q=$ Write Input Queue, $R Q=$ Read Input Queue, $X=$ Crossbar, and $E C C=$ Error Correction Code. This paper focuses on the LA, RE, and $G A$ stages of the pipeline.

for re-arbitration once all flits of a packet are delivered via the port.

- Virtual Cut-Through Routing. The 21364 uses virtual cutthrough routing in which flits of a packet proceed through multiple routers until the header flit gets blocked at a router. Then, all flits of the packet are buffered at the blocking router until the congestion clears. Subsequently, the packet is scheduled for delivery through the router to the next router and the same pattern repeats. To support virtual cut-through routing, the 21364's router provides buffer space for 316 packets per input port [26]. Note that a packet is never dropped from the network in the absence of errors.

- Adaptive Routing in the Minimal Rectangle. In the 21364, packets adaptively route within the minimum rectangle. Given two points in a torus (in this case, the current router and the destination processor), one can draw four rectangles that contain these two points as their diagonally opposite vertices. The minimum rectangle is the one with the minimum diagonal distance between the current router and the destination. Thus, the adaptive routing algorithm has to pick one output port among a maximum of two output ports that a packet can route in. Packets that follow adaptive routing may not be delivered in order, but the coherence protocol in 21364 is designed to handle out of order traffic.

- Virtual Channels. The 21364 router uses virtual channels [9] to break deadlocks in the coherence protocol and the routing algorithms. It assigns a virtual channel group to each coherence packet class. By design, these virtual channel groups are ordered, such that a request packet can never block a block response packet. Each group (except the special class) contains three virtual channels: adaptive, VC0, and VC1. Packets adaptively route within the adaptive channel until they get blocked. ${ }^{2}$ Blocked packets are then routed in the

2 This is only true for non-I/O packets. Read and Write I/O packets only route in the deadlock-free channels to adhere to the Alpha 21364's I/O ordering rules. 


\begin{tabular}{|l|l|l|l|l|l|l|l|}
\hline & G-N & G-S & G-E & G-W & G-L0 & G-L1 & G-I/O \\
\hline L-N rp0 & & & & & & & \\
\hline L-N rp1 & & & & & & & \\
\hline L-S rp0 & & & & & & & \\
\hline L-S rp1 & & & & & & & \\
\hline L-E rp0 & & & & & & & \\
\hline L-E rp1 & & & & & & & \\
\hline L-W rp0 & & & & & & & \\
\hline L-W rp1 & & & & & & & \\
\hline L-Cache rp0 & & & & & & & \\
\hline L-Cache rp1 & & & & & & & \\
\hline L-MC0 rp0 & & & & & & & \\
\hline L-MC0 rp1 & & & & & & & \\
\hline L-MC1 rp0 & & & & & & & \\
\hline L-MC1 rp1 & & & & & & & \\
\hline L-I/O rp0 & & & & & & \\
\hline L-I/O rp1 & & & & & \\
\hline
\end{tabular}

Figure 5. The 21364 router's connection matrix. This figure represents the router's crossbar connections in a matrix format " $G$ - $X$ " denotes output port arbiter for output port $X$. " $L-X$ rpY" denotes input port arbiter for input port $X$ and read port $Y$. The shaded boxes represent no connection.

deadlock-free channels, $\mathrm{VC} 0$ and $\mathrm{VC} 1$, which follow strict dimension-order routing. Duato [13] has shown that such a scheme breaks routing deadlocks in such networks. Because of virtual cut-through routing, however, packets can return from the deadlock-free channels to the adaptive channel. For performance reasons, the adaptive channels have the bulk of the packet buffers, whereas the VCO and VC1 typically have one or two buffers. In the 21364 there is a total of 19 virtual channels (three for each of the six non-special coherence classes and one for the special class).

- Ports. Each port is 39 bits wide to match the network's flit size. Each router has eight input ports and seven output ports. The input ports include four 2D torus ports (north, south, east, and west), one cache port (that sends cache miss requests, etc.), two memory controller ports (that sends responses to cache miss requests), and one $\mathrm{I} / \mathrm{O}$ port. The buffers at each input port have two read ports to allow the arbitration algorithm greater choice in matching inputs to outputs. Like the input ports, the output ports are divided into four 2D torus ports (north, south, east, and west), two memory controller ports, and one I/O port. Inside the processor, the two memory controller ports are also tied to the internal cache and, hence, there is no separate explicit cache output port.

\subsection{Router Pipeline}

The 21364's router has nine pipeline types based on the input and output ports. There are three types of input and output ports: local (cache and memory controllers), interprocessor (off-chip network), and I/O. Any type of input port can route packets to any type of output port, leading to nine types of pipeline. Figure 4 shows two such pipeline types.

As Figure 4 shows, the router pipeline in the 21364 consists of several stages that perform router table lookup, decoding, arbitration, forwarding via the crossbar, and ECC calculations. A packet originating from the local port looks up its routing information from the router table and loads it up in its header. The decode stage decodes a packet's header information and writes the relevant information into an entry table, which contains the arbitration status of packets and is used in the subsequent arbitration pipeline stages.
The 21364's arbitration pipeline, which is the focus of this paper, consists of three stages: LA (input port arbitration), RE (Read Entry Table and Transport), and GA (output port arbitration). ${ }^{3} \quad$ The input port arbitration stage finds packets from the input buffers and nominates one of them for output port arbitration. Each input buffer has two read ports and each read port has an input port arbiter associated with it. Thus, the 21364 has a total of 16 input port arbiters. The input port arbiters perform several readiness tests, such as determining if the targeted output port is free, using the information in the entry table.

The output port arbiters accept packet nominations from the input port arbiters and decide which packets to dispatch. Each output port has one arbiter, so the 21364 has a total of seven output port arbiters. Once an output port arbiter selects a packet for dispatch, it informs the input port arbiters of its decision, so that the input port arbiters can re-nominate the unselected packets in subsequent cycles.

Figure 5 shows the crossbar connection between the input and output port arbiters. Although the connections form a crossbar between input and output ports, the individual read ports are not connected to all the output ports. The same crossbar connection is followed by the datapath in the $\mathrm{X}$ stage of the pipeline (Figure 4).

In addition to the basic pipeline latency, there are six additional delay cycles along the path of a packet, including synchronization delay, pad receiver and driver delay, and transport delay from the pins to the router and from the router back to the pins. Thus, the on-chip pin-to-pin latency from a network input to a network output is 13 cycles. At $1.2 \mathrm{GHz}$, this leads to a pin-to-pin latency of 10.8 nanoseconds.

Also, the network links that connect the different 21364 chips run at $0.8 \mathrm{GHz}$, which is $33 \%$ slower than the internal router clock. The input port arbitration internally nominates packets at the appropriate cycles so that packets leaving the router are synchronized with the off-chip network clock.

\section{ARBITRATION ALGORITHMS}

In the 21364 router, the 16 input port arbiters and 7 output port arbiters work together to implement the arbitration algorithm. The 21364 router's arbitration problem can be modeled in two ways. First, it can be modeled as a matching problem in a bipartite graph with 16 input port arbiters and 7 output port arbiters. Each connection between the input and output port arbiters will carry a certain "weight." Then, a Maximum Weight Matching (MWM) algorithm, will try to find a match that maximizes the total weight of the connections selected by the match. Examples of such MWM algorithms are LQF (longest queue first), which uses the number of waiting packets at an input port as the weight for a connection, and OCF (oldest cell first), which uses the waiting time for the oldest packet at an input buffer as the weight for a connection [24]. Unfortunately, the MWM algorithms require $\mathrm{O}\left(\mathrm{N}^{3}\right)$ iterations in the worst case [25], which makes it very difficult to implement them in hardware in a few cycles. Also, approximations of the MWM algorithm, such as RPA [1], MUCS [12], Laura and Serena [25], and Apsara [18], are also not implementable in hardware within a few cycles.

\footnotetext{
${ }^{3}$ The 21364 router's input and output port arbiters are also referred to as local arbiters (LA) and global arbiters (GA), respectively [26].
} 
In this paper, as an upper bound we use an algorithm called the Maximal Cardinality Matching Algorithm (MCM), which is basically MWM with all connections having equal weights. MCM exhaustively searches the space for the maximum number of matches between input and output port arbiters. We use MCM only in our non-timing simulations because we do not know how to implement MCM in hardware within a few cycles.

Another way to model the arbitration problem is to use a two-dimensional "connection" matrix with input ports forming the rows and output ports forming the columns. Such a representation makes it easier for us to explain the arbitration algorithms we study in this paper. In this representation, an input port nominates packets to output ports by filling up the corresponding row in the matrix. An output port chooses packets from input ports by scanning the corresponding column in the matrix.

Figure 5 shows the connection matrix for the 21364 router. Given this representation, an arbitration algorithm for the 21364 router then needs to answer the following questions:

- Which packets should an input port arbiter nominate to an output port arbiter? An input port arbiter can pick packets out of all the buffers in each of the 19 virtual channels. For correctness and improved performance, each input port arbiter (independent of the arbitration algorithm) obeys some basic constraints, such as whether the corresponding output port is free to dispatch a packet. Each input port arbiter then selects the oldest packet, which satisfies the basic constraints, from the least-recently selected virtual channel. An input port arbiter fills up the corresponding row in the connection matrix with the packets it selects.

- Can the same packet be nominated multiple times? Any packet can proceed along a maximum of two directions because 21364 adaptively routes packets within the minimal rectangle (Section 2.1). Thus, a packet can be nominated to at most two output port arbiters. Multiple nominations have the advantage that a packet would have a greater probability of being dispatched in the same cycle. However, multiple nominations of the same packet also imply extra interaction between input and output ports to ensure that the same packet is not dispatched through two different output ports.

- Which packets should an output port arbiter pick from the packets nominated to it by the input port arbiters? An output port arbiter examines its corresponding column for packets nominated to it by all the input port arbiters. Then, to select a packet from a column it can use a variety of policies, such as random [11], round-robin [31], least-recently selected [35], some kind of a priority chain [10], or the "Rotary Rule." Such prioritization policies are easily implemented in hardware via a priority matrix. We describe the implications of some of these policies later in this section. Section 3.4 describes the Rotary Rule in detail.

- Can there be multiple iterations (or passes) through the matrix? Multiple iterations through the nomination and selection procedure allow the arbitration algorithm to find more matches compared to a single iteration. However, multiple iterations would also incur higher number of cycles to perform the arbitration.

Answers to the above questions have important implications on the hardware implementation of an arbitration algorithm. For example, these choices determine how much synchronization is required among all the 23 arbiters (16 for input, 7 for output) in the router and whether the arbitration algorithm can be effectively pipelined.

The rest of this section discusses how PIM, WFA, and SPAA answer the above questions (Sections 3.1, 3.2, and 3.3). Section 3.4 describes the Rotary Rule and how it can be incorporated into WFA and SPAA.

\subsection{Parallel Iterative Matching}

The Parallel Iterative Matching (PIM) algorithm, proposed by Anderson, et al. [2], was designed to quickly identify a set of conflict-free packets for transmission through an ATM switch. PIM works extremely well in such ATM switches where the matching algorithm may be implemented in software. The key to PIM's success lies in its interaction between input and output port arbiters, which avoids arbitration collisions incurred by naïve algorithms, such as OPF (Figure 2).

Below we describe the algorithm's three key steps for the 21364 router:

1. Nominate. Each unmatched input port arbiter nominates a packet for each output port arbiter for which it has a packet. The same packet can be nominated to multiple output port arbiters.

2. Grant. If an unmatched output port arbiter receives any requests, it accepts one randomly and informs the corresponding input port arbiter of its decision.

3. Accept. If an input port arbiter receives grants for multiple output port arbiters, it selects one randomly.

PIM iterates over the above three steps until the algorithm converges. According to Anderson, et al. [2], PIM usually converges within $\log _{2} \mathrm{~N}$ iterations, so the 21364 router would need four iterations ( $\mathrm{N}=16$ input port arbiters) of the above three steps. Researchers have proposed variations of PIM, such as iSLIP [23] that can be implemented in hardware, but their matching capabilities are similar to PIM's.

PIM has two properties that make it difficult to implement in hardware in a few cycles. First, it can nominate the same packet to multiple output port arbiters, even though multiple output port arbiters cannot dispatch the same packet. PIM avoids multiple dispatches using an additional synchronization step (Step 3) between the input and output port arbiters. Unfortunately, this synchronization makes it difficult for input port arbiters to nominate other packets until they receive their grants from the output port arbiters. In other words, it is hard to do input port arbitrations in consecutive cycles, which makes it difficult to pipeline PIM.

Second, PIM requires multiple iterations of its three steps. This would further increase the delay of the algorithm. Hence, we use a variant of PIM called PIM1, which is PIM with one iteration of its three steps. McKeown has shown, however, that PIM1's matching capabilities are significantly worse than PIM's [24].

In our evaluation, we assume that PIM1 takes four cycles for arbitration. We would implement PIM1 using a centralized $16 \times 7$ connection matrix, which receives inputs from the input port arbiters and lets output port arbiters iterate over its columns. The first three cycles of the four cycles consist of matrix operations and wire delays: 1.5 cycles to select packets from the input ports and load up the matrix (i.e., nominate) and 1.5 cycles to grant and accept (via passes over the matrix). The speed at which the matrix can be evaluated is limited because of 


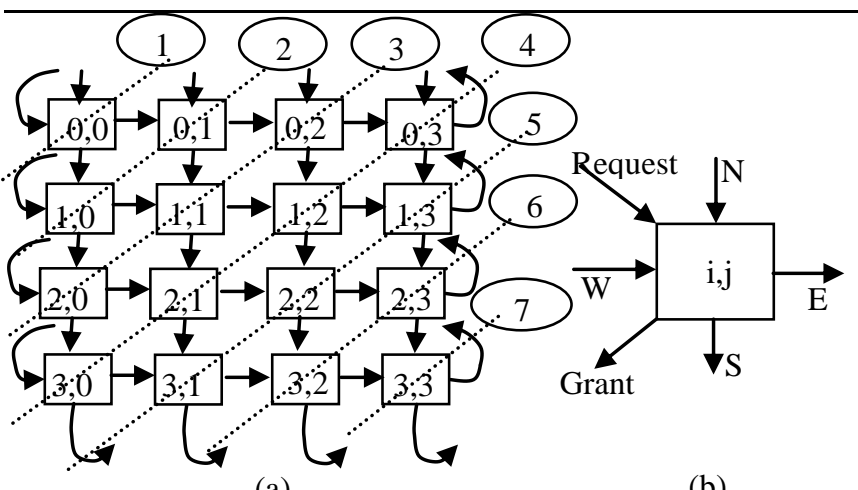

Figure 6. Operations of the Wave-Front Arbiter (WFA) for a $4 \times 4$ connection matrix. (a) The dotted lines (with circled numbers) show the wave fronts. Each square represents an arbitration cell with coordinates $i, j . \quad$ (b) This figure shows an arbitration cell ( $i, j)$ of the WFA matrix.

dependences between the input and output ports and the limited number of logic levels (12-13) per cycle in our process technology. Further, PIM1 requires a random number generator, which will require a few additional logic levels.

In this implementation of PIM1, a new arbitration can be started every three cycles because nominate, grant, and accept take a total of three cycles. Starting a grant or accept before the prior nominate step is difficult because of two reasons. First, the total nominations for the matrix could be up to 54 (unshaded boxes in Figure 5). Keeping track of these large number of inflight packets (i.e., nominated, but not delivered) would require additional state and could increase the number of cycles incurred by PIM1. Second, we would have to maintain multiple copies of the matrix to act as buffers for the pipeline stages for the arbiters. These matrices must be consistent with one another and should not be loaded with stale packets. Again, this may further increase the number of cycles incurred by PIM1.

The fourth cycle of PIM1's four-cycle arbitration accounts for wire delays from the matrix to the output ports and can be pipelined.

\subsection{Wave-Front Arbiter}

Tamir and Chi [36] proposed the Wave-Front Arbiter (WFA) for routers in interconnection networks. WFA has been implemented in the SGI Spider interconnect [16]. WFA is a much lighter-weight algorithm than PIM1 and could be used effectively in routers that operate at a much lower frequency than that of the 21364 router. For example, WFA in the Spider switch operates at $100 \mathrm{MHz}$ and is implemented within a single 10 nanosecond clock cycle. Also, the key to WFA's success lies in its interaction among the input port arbiters and among the output port arbiters, which allows it to avoid arbitration collisions that may be incurred by naïve algorithms, such as OPF (Figure 2).

WFA operates on the entire connection matrix as a whole. First, the input port arbiters load up the matrix with their nominations. Then, evaluation of the matrix starts from a specific cell in the matrix. The evaluation proceeds in a wave front as follows (Figure 6):

$$
\begin{aligned}
& \text { Grant }_{i, j}=\text { Request }_{i, j} \text { and } N_{i, j} \text { and } W_{i, j} \\
& S_{i, j}=N_{i, j} \text { and } \operatorname{NOT}\left(G \operatorname{Grant}_{i, j}\right) \\
& E_{i, j}=W_{i, j} \text { and NOT }\left(G_{i, j}\right)
\end{aligned}
$$

The connection matrix is represented in hardware as a twodimensional array of arbitration cells. The position of each cell in the connection matrix is denoted by i,j. Request denotes that an input port arbiter has nominated a packet for that arbitration cell. Grant denotes that the specific arbitration cell has been chosen for packet delivery. Then, following the above equations, no other cell in the same row (i.e., same input port arbiter) and no other cell in the same column (i.e., same output port arbiter) as the granted cell, would select any other packet for dispatch. Also, note that $\mathrm{N}_{\mathrm{i}, \mathrm{j}}=\mathrm{S}_{\mathrm{i}-1, \mathrm{j}}$ and $\mathrm{W}_{\mathrm{i}, \mathrm{j}}=\mathrm{E}_{\mathrm{i}, \mathrm{j}-1}$.

Thus, as Figure 6 shows, if the evaluation starts with wavefront 1 , then the cell $(0,0)$ will be evaluated first, followed by the cells $(0,1)$ and $(1,0)$, which make up wavefront 2 . Subsequent wavefronts will be evaluated in this fashion.

To ensure fairness, the first cell from where the wave fronts begin must be chosen carefully. Tamir and Chi suggested using a robin-robin scheme to choose the first cell. We will refer to this scheme as WFA-base. Section 3.4 will show how to use the Rotary Rule to choose the first cell, which we will refer to as WFA-rotary.

Although the WFA is very appealing, it is not amenable to efficient pipelining. This is because input port arbiters in WFA, like in PIM, can nominate the same packet to multiple output port arbiters. PIM uses synchronization between input and output port arbiters (Step 3 in PIM) to avoid multiple dispatches of the same packet. In contrast, WFA requires communication between the output port arbiters-via the propagation of the $\mathrm{N}$ and $\mathrm{S}$ signals along the columns - to avoid dispatching the same packet through multiple output port arbiters. Note that WFA uses the same mechanism-i.e., interaction among output port arbiters-to avoid arbitration collisions and, thereby, provide good matching performance. Thus, interaction between output port arbiters is fundamental to the WFA algorithm. Additionally, micropipelining the matrix operations themselves-by pipelining the "waves" of the WaveFront Arbiter-is difficult because the starting cell (as indicated in the last paragraph) changes every cycle.

We assume a four-cycle arbitration delay for the WFA. Our timing is optimistically based on the Wrapped Wave-Front Arbiter, proposed by Tamir and Chi. The Wrapped WFA provides matching performance similar to that of WFA's, but executes faster in hardware by starting multiple wavefronts in parallel. As in PIM1, the first three cycles of WFA's four-cycle arbitration are spent on matrix operations and wire delays: 1.5 cycles to nominate packets and load up the matrix and 1.5 cycles to evaluate the matrix. WFA suffers from the same problems as PIM1 (Section 3.1) and hence a new arbitration can only be restarted every three cycles. Again, as in PIM1, the fourth cycle accounts for wire delays from the matrix to the output ports and can be pipelined.

\subsection{Simple Pipelined Arbitration Algorithm}

The Simple Pipelined Arbitration Algorithm (SPAA) implemented in the 21364 carefully minimizes the impact of features, such as interaction between input and output ports, which would be hard to pipeline. However, this also makes its matching performance much worse than PIM1 and WFA because it may not be able to avoid arbitration collisions (Figure 2), particularly in the presence of a large number of free output ports. Thus, in terms of its matching capability, SPAA is more like OPF from Figure 2. 
Like PIM, SPAA has three steps:

1. Nominate. Each input port arbiter nominates a packet for only one output port arbiter, if there is one. A nominated packet cannot be nominated again in subsequent cycles until Step 3 of this algorithm completes.

2. Grant. If an output port arbiter receives multiple requests, it selects the packet from the least-recently selected input port arbiter. Then, it informs all input port arbiters connected to it of its decision.

3. Reset. An input port arbiter resets the state of all nominated packets that are not selected by the output port arbiter, so that they can be nominated again.

SPAA has three important properties that make it amenable to easy hardware implementation and pipelining. First, unlike PIM1 or WFA, an input port arbiter nominates a packet to only one output port arbiter. This avoids the extra interaction required between the input and output port arbiters (as in PIM1) or between the output port arbiters (as in WFA). Nevertheless, because a pair of input port arbiters shares the same set of input buffers via different read ports, the input port arbiters in a pair must synchronize to ensure that they do not choose the same packet. However, because the synchronization is between pairs of input port arbiters located in close proximity, this is not hard to implement.

Nominating a packet to only one output port also has the added benefit that a packet can be speculatively read out from an input buffer as soon as it is nominated by an input port arbiter (but before the output port arbitration is complete), much like the way direct-mapped caches allow processors to speculatively read out data before the address comparison completes [20]. Of course, the read is wasted if the output port does not select the specific packet that was speculatively read out.

Second, SPAA can be implemented as a distributed router with the input and output port arbiters sitting right next to their corresponding ports. In contrast, because of PIM1 and WFA's high level of interaction between input and output ports, it is easier to implement PIM1 and WFA using a centralized connection matrix. The distributed implementation of SPAA allows it to directly send input port nominations from the input to the output ports without an intervening connection matrix. This helps reduce the number of cycles incurred by SPAA.

Third, SPAA need only maintain a small list of in-flight packets - that is, only 16-because each input port can only nominate a maximum of one packet. In contrast, aggressive and more complicated implementations of PIM1 and WFA would have required us to maintain state for 54 in-flight packets, which would complicate their implementation. SPAA's small number of in-flight packets (i.e., nominated from the input port, but not yet accepted by the output port) facilitates effective pipelining of SPAA. Thus, unlike PIM1 and WFA, new input port arbitrations in SPAA can be restarted every cycle.

Thus in summary, SPAA incurs only three cycles (Figure 4) for its arbitration compared to the four cycles required by PIM1 or WFA and SPAA can be pipelined effectively, so that an input port arbitration can be started every cycle. SPAA's three cycles consist of input port arbitration (i.e., nominate), transport from input to output port, and output port arbitration (Figure 4).

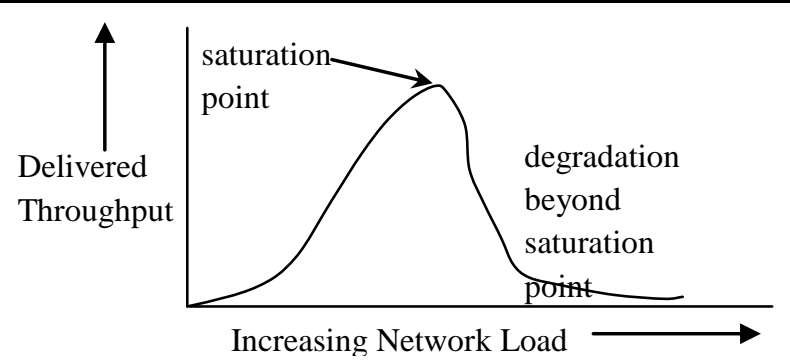

Figure 7. Possible network behavior with increasing network load. Network performance degrades rapidly beyond the saturation point.

SPAA's Step 2 (Grant) selects packets based on the leastrecently selected policy. We call this SPAA-base. In the next subsection, we discuss how SPAA can use the rotary rule to select an input port arbiter. We call this SPAA-rotary.

\subsection{Rotary Rule}

Under extremely heavy loads most multiprocessor networks suffer from tree saturation [28][30], which can dramatically degrade a network's performance beyond the saturation point (Figure 7). Such tree saturation occurs when multiple packets contend for a single resource (e.g., a link between nodes) creating a hot spot. Since only one packet can use the resource, other packets must wait. These waiting packets occupy buffers and thus delay other packets, even though they may be destined for a completely different node and share only one link on their paths to their respective destinations. This process continues and waiting packets delay other packets producing a tree of waiting packets that fans out from the original hot spot. Eventually, this clogs the network bringing down the delivered throughput of the entire network.

The 21364 network is no exception and can get saturated at extremely high load levels. Interestingly, the network produces a cyclic pattern of network link utilization with extremely high levels of uniform random input traffic. This is because as the network gets saturated, it puts backpressure on the links in the tree. Eventually, this backpressure throttles the routers in the tree and forces them to avoid injecting new traffic, which causes the network congestion to clear up slowly. The period of this cycle increases with the diameter of the network because it takes longer to fill up the buffers on the path and propagate the backpressure.

Ideally, we would like network throughput to remain at the same level as exhibited at the saturation point, instead of degrading dramatically beyond the saturation point. Most proposed solutions rely on throttling the input network load based on some estimate of congestion, so that the network never goes beyond the saturation point. Lopez, et al. [21][22] use the number of busy virtual channels in a router to estimate congestion. Baydal, et al. [4] proposes an approach that counts a subset (free and useful) of virtual channel buffers to decide whether to throttle or not. Other researchers (e.g., [34], [30], [38]) have proposed the use of a variety of global heuristics to determine congestion.

Fortunately, the 21364 network has two properties that limit the network load. First, a 21364 processor can have only 16 outstanding cache miss requests to remote memory or caches. This limits the load the 21364 network can observe.

Second, the 21364 is a "direct" network in which the same router is responsible for both new traffic (originating from the 
local ports, such as the cache port or memory controller ports) and cross-traffic between routers. Thus, prioritizing the crosstraffic over new traffic generated from the local ports has the beneficial effect of both clearing the network congestion as well as throttling the input load into the network. We call this prioritization policy the "Rotary Rule." The name is derived from the Massachusetts rotaries in which vehicles in the rotary has higher priority to exit than vehicles trying to enter the rotary.

It is easy to implement the Rotary Rule for PIM1, WFA, and SPAA. In PIM1 and SPAA, the output port arbiters would select packets nominated by the input port arbiters for the network ports before they select packets from the local ports. Within the network ports, we use least-recently used selection policy. In WFA, the selection of the first cell to start the arbitration process would follow the Rotary Rule. Thus, cells connected to the input port arbiters for the network ports would get the highest priority to be the first cell from where the wavefronts will start. In this paper, we only evaluate the Rotary Rule for WFA and SPAA. We call these variants WFA-rotary and SPAA-rotary, respectively.

The Rotary Rule's prioritization of cross-traffic packets can create starvation in the network. The 21364 router implements an anti-starvation algorithm for certain corner cases. The Rotary Rule simply relies on this anti-starvation algorithm to clear any starvation caused by its prioritization policy. The anti-starvation algorithm assigns two different colors to packets waiting at a router: an old color and a new color. If the number of old colored packets exceed a threshold, the 21364 ensures that all the old colored packets are drained before any new colored packets are routed. Further discussion of the antistarvation algorithm is beyond the scope of this paper.

The 21364 network provides the Rotary Rule as an optional mode programmable at boot time. It is an optional mode because we believe most applications will not stress the network to the extent of pushing it into saturation. Nevertheless, we provide it both as a "safety net" for the 21364 processor and as a mechanism that may have allowed its use in future processors with many more outstanding misses (e.g., the next generation Alpha 21464 processor would have had 64 outstanding misses).

\section{METHODOLOGY}

This section describes our performance model, traffic patterns, and performance metric.

\subsection{Performance Model}

Our evaluation of the 21364's arbitration algorithm choices is based on two kinds of performance models written in the Asim framework [15], unlike Bhuyan [5] or Peh and Dally [27], who had used analytical modeling to understand the behavior of arbiters and routers ${ }^{4}$. Our first model-what we call the standalone model-allows us to evaluate the matching capabilities of MCM, PIM, PIM1, WFA, and SPAA in a single 21364 router (just like a cache simulator would allow one to evaluate the cache miss ratio without any timing information).

Our second model — what we call the timing model-is an extremely detailed performance model of the 21364 router. We

\footnotetext{
4 Bhuyan's paper pre-dates PIM1 and WFA. Peh and Dally focused on developing analytical models for router pipelines, but did not compare the performance of different arbitration algorithms.
}

have validated this model against a production-level performance model of the 21364 network architecture. We have modeled the detailed timing characteristics of PIM1, WFAbase, WFA-rotary, SPAA-base, and SPAA-rotary using this timing model.

We described most of the parameters of the timing runs in Section 2.2 and Section 3. In addition, we assume 73 nanoseconds for the memory system's response time, 25 cycles for the on-chip L2 cache's response time, and 3 network clocks (running at $0.8 \mathrm{GHz}$ ) for latency on each network link. Most of the results we present in this paper are for a 16-processor $(4 \times 4)$ network and a 64-processor $(8 \times 8)$ network. Although the 21364 network only scales up to 128 processors, Section 5.3 examines results for a 144-processor (12x12) network to understand how the arbitration algorithms may scale for larger network configurations.

\subsection{Traffic Patterns}

We evaluate our timing models using a mix of synthetic traffic patterns as opposed to real workloads. Simulations of real workloads, such as database servers, would have helped us make more accurate predictions about the performance impact of the different arbitration algorithms. Such simulation, however, would have required complex full-system simulation (including the operating system), which our modeling infrastructure is unable to handle today. Trace-driven simulation would have been an alternative, but that also has its limitations [7].

Nevertheless, synthetic workloads have two advantages. First, they often tend to increase the contention for resources for sub-optimal/worst case performance scenarios [39]. Second, they represent communication patterns in many real-world applications [14].

Recently, Towles and Dally [39] demonstrated a technique to construct synthetic traffic patterns that result in worst-case performance for oblivious routers. Unfortunately, there is no known similar technique for adaptive routers, such as the 21364.

Our synthetic patterns can be defined along two dimensions. The first dimension selects the mix of coherence packets. We use $70 \%$ two coherence hop transactions (3-flit request and a 19-flit block response) and 30\% three coherence hop transactions (3-flit request, 3-flit forward, and a 19-flit block response) to model a mix of coherence traffic. We, however, ignore traffic generated from cache replacements or invalidations to make our analysis simpler. ${ }^{5}$ Note that a coherence hop only specifies a single packet, which can take multiple router hops via the network.

The second dimension selects the destination of the requests and forwards. We use three patterns for such selection: uniform, bit-reversal, and perfect-shuffle. If the bit-coordinate of the source processor can be represented as $\left(a_{n-1}, a_{n-2}, \ldots a_{1}, a_{0}\right)$, then the destination bit-coordinates for bit-reversal and perfectshuffle are $\left(a_{0}, a_{1}, \ldots, a_{n-2}, a_{n-1}\right)$ and $\left(a_{n-2}, a_{n-3}, \ldots, a_{0}, a_{n-1}\right)$ respectively.

\subsection{Performance Metric}

We use the Burton Normal Form (BNF) [14] to express the performance of our different arbitration algorithms. A BNF graph uses observed latency as its vertical axis and delivered

\footnotetext{
${ }^{5}$ The 21364 processor can have 16 outstanding cache replacement requests.
} 


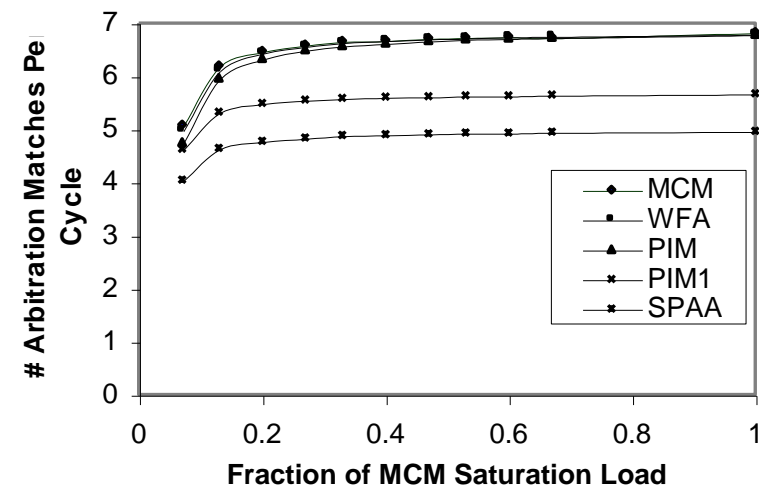

Figure 8. Standalone comparison of matching capabilities of different arbitration algorithms for a single 21364 router with increasing router load for zero output port occupancy. The horizontal axis plots the input router load as a fraction of the load required to saturate MCM.

throughput as its horizontal axis. For our BNF graphs, we use the average latency of a packet through the network as the vertical axis. The minimum per-packet latency with a $4 \times 4$ network, uniform random distribution of destinations, and a 70/30 mix of 2-hop and 3-hop coherence transactions is about $45 \mathrm{~ns}$ (nanoseconds). The $45 \mathrm{~ns}$ can be broken into $2.5 \mathrm{~ns}$ of local port latency, 34 ns of network transit latency for the first flit, and 8.5 nanoseconds of latency for the rest of a packet. The last number is averaged across the different packet sizes for our coherence transaction mixes.

We represent the delivered throughput as flits/router/ns (where $\mathrm{ns}=$ nanoseconds). The maximum throughput is two flits/router/cycle because the 21364 router has two local ports to sink packets and only one flit can be delivered to a local port per cycle. Thus, the maximum delivered throughput can be 2.4 flits/router/nanosecond (= $2 / 0.83)$. In reality, however, the actual delivered throughput will be significantly lower because the network links are $33 \%$ slower than the processor and the network links often carry cross-traffic, whose residence time in the network increases with the size of the network.

We ran each timing simulation for 75,000 cycles. We have validated that simulation for this number of cycles is sufficient to predict the steady-state behavior of the network.

\section{RESULTS}

This section presents our standalone, timing, and scaling results. Although we present our results only for a subset of the network sizes and traffic patterns, our results are qualitatively similar across a wide spectrum of the design space.

\subsection{Standalone Results}

Figure 8 shows that when all output ports are free MCM, PIM, and WFA are indeed superior arbitration algorithms compared to PIM1 and SPAA. We generated Figure 8 by loading up a single router with input packets and using the following assumptions:

- All arbitration algorithms take one cycle to execute.

- All output ports are free at the time of the arbitration.

- $50 \%$ of the traffic is local and destined for the local memory controller and I/O ports. The rest are destined uniformly for the other network ports.

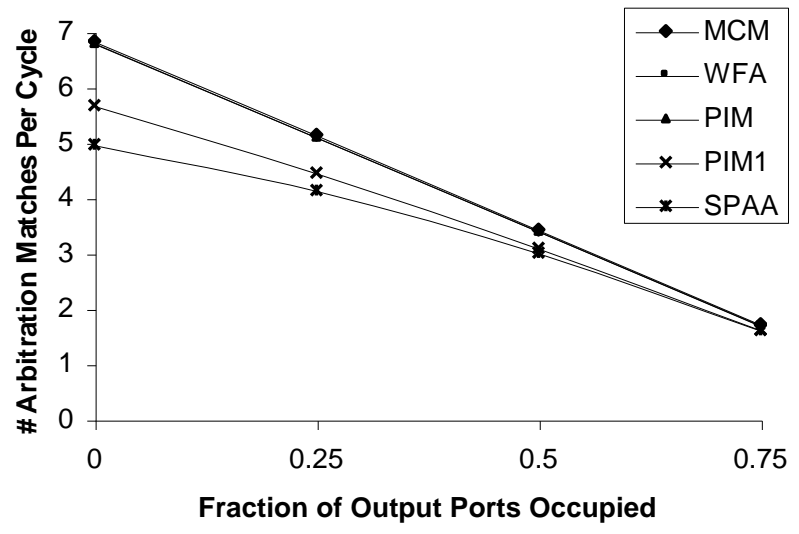

Figure 9. Standalone comparison of matching capabilities of different arbitration algorithms for a single 21364 router with increasing output port occupancy at the MCM saturation load.

- The number of arbitration matches is averaged across 1000 iterations of the arbitration algorithms. Because the traffic is generated randomly, in some cases even MCM, which exhaustively searches for the maximum number of matches, is not able to find a perfect match. However, the number of matches found by MCM is usually very close to the maximum, i.e., seven (because there are seven output ports).

- Although all algorithms execute in one cycle, they all follow the basic 21364 router constraints, such as adaptive routing within the minimum rectangle.

Under the above assumptions, the number of matches found by WFA and PIM are almost close to that found by MCM. PIM1 does slightly worse and SPAA is the worst. At the MCM saturation load, the number of matches found by MCM, WFA, and PIM are $36 \%$ higher than that found by SPAA. PIM1's number of matches is $14 \%$ higher than SPAA's.

Figure 9, however, shows that under realistic conditions the difference between the various arbitration algorithms reduces dramatically. Figure 9 plots the arbitration matches per cycle for the different algorithms for various levels of output port occupancy. In the 21364 router, packet sizes range from 3 to 19 flits, so when a packet wins an arbitration, it occupies an output port for several cycles. It is unnecessary to arbitrate for an output port while it is busy delivering a packet. As the fraction of occupied output ports increases, the difference between the algorithms reduces and completely disappears when $75 \%$ of the output ports are occupied.

Thus, under heavy loads (when output port occupancy is likely to be high), it does not matter which arbitration algorithm we choose. Rather, it is better to choose an algorithm that is more suited to faster implementation. We designed SPAA based on this observation.

Another way to look at these results is that WFA and PIM's matching capabilities are more suited to routers with significantly higher number of ports compared to what we have in the 21364 router.

\subsection{Timing Results}

Figure 10 shows the performance of the five arbitration algorithms-PIM1, WFA-base, WFA-rotary, SPAA-base, and SPAA-rotary-for different network sizes and traffic patterns. SPAA-base significantly outperforms both PIM1 and WFAbase, which perform similarly. For example, in the $4 \times 4$ 

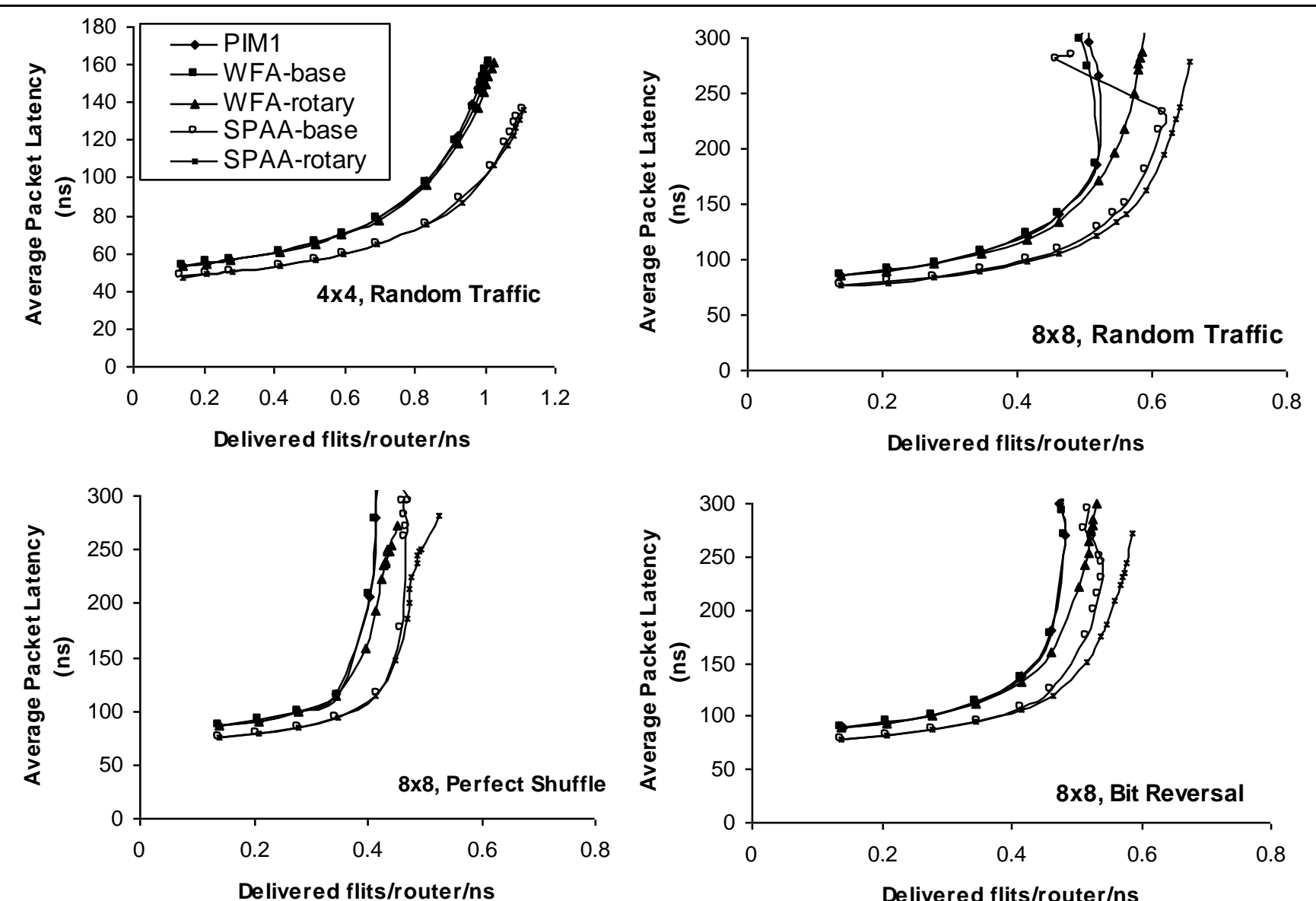

Figure 10. This figure shows the performance of the 21364 network with different arbitration algorithms, network sizes (4x4 and 8x8), and traffic patterns (Random, Bit Reversal, and Perfect Shuffle).

network, with random traffic SPAA-base provides about $11 \%$ higher throughput compared to PIM1 and WFA-base when the average packet latency is about 83 nanoseconds. Similarly, in the $8 \times 8$ network, with random traffic SPAA-base provides about $24 \%$ higher throughput compared to PIM1 and WFAbase when the average packet latency is about 122 nanoseconds. The results for bit reversal and perfect shuffle for the $8 \times 8$ network are qualitatively similar.

Figure 10 also shows that the Rotary Rule prevents both WFA-rotary and SPAA-rotary from performance degradation under heavy network loads. The $4 \times 4$ network does not show saturation behavior, so the performance of WFA-base and SPAA-base are similar to WFA-rotary and SPAA-rotary respectively. In the $8 \times 8$ network, WFA-base and SPAA-base perform similar to WFA-rotary and SPAA-base respectively, until the network hits the saturation point. Thereafter, the delivered throughput of both WFA-base and SPAA-base degrade rapidly, while WFA-rotary's and SPAA-rotary's delivered throughputs continue to increase.

Thus, at about an average packet latency of 280 nanoseconds, WFA-rotary improves throughput by $16 \%$ over WFAbase and SPAA-rotary improves throughput by $43 \%$ over SPAA-base. Note that WFA-base shows less performance degradation compared to SPAA-base. We suspect this happens because the interaction between WFA's output port arbiters (unlike in SPAA) makes the worst case behavior of WFA-base better than that of SPAA-base.
Finally, pipelining provides SPAA a significant boost in performance compared to PIM1 and WFA (not shown here). For example, if we could implement WFA as a three-cycle arbitration mechanism like SPAA, then pipelining is the key difference between WFA and SPAA. In an 8x8 network, with random traffic SPAA provides a throughput boost of about $8 \%$ compared to such a configuration of WFA-base with 122 nanoseconds of average packet latency. This shows pipelining the arbitration mechanism does help SPAA's performance.

\subsection{Scaling Results}

This section studies the performance of PIM1, WFA-rotary, and SPAA-rotary under three different scaling conditions: with twice the router pipeline length as in the 21364 router, with higher input load than 21364 can offer, and with a bigger network. The router pipeline length could potentially double in future generations, given the scaling trends of technology today. Figure 11a shows the results for PIM1, WFA-rotary, and SPAA-rotary for a pipeline two times longer than and running at twice the frequency of the 21364 router's pipeline. The arbitration latencies for PIM1, WFA-rotary, and SPAA-rotary are 8,8 , and 6 cycles respectively. As the figure shows, SPAA-rotary performs significantly better with longer pipelines because SPAA-rotary is pipelined, unlike the other two arbitration algorithms. Thus, for example, at about 100 nanoseconds of average packet latency, SPAA-rotary provides greater than $60 \%$ higher throughput compared to PIM1 and WFA-rotary. 


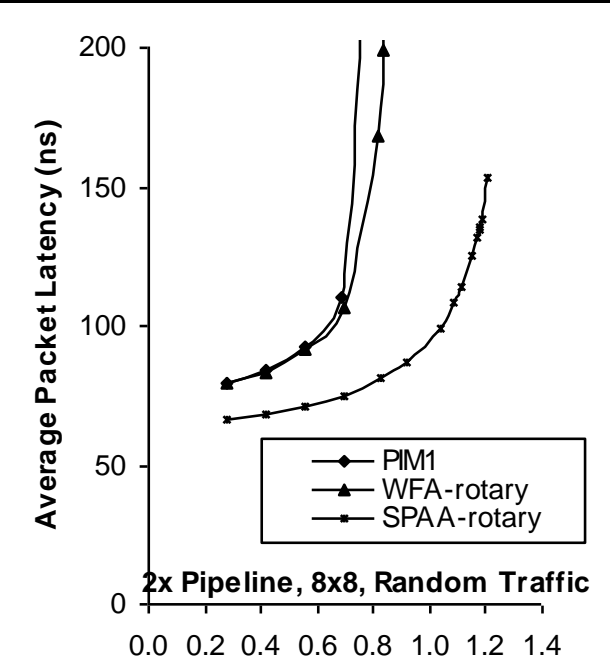

Delivered flits/router/ns

(a)

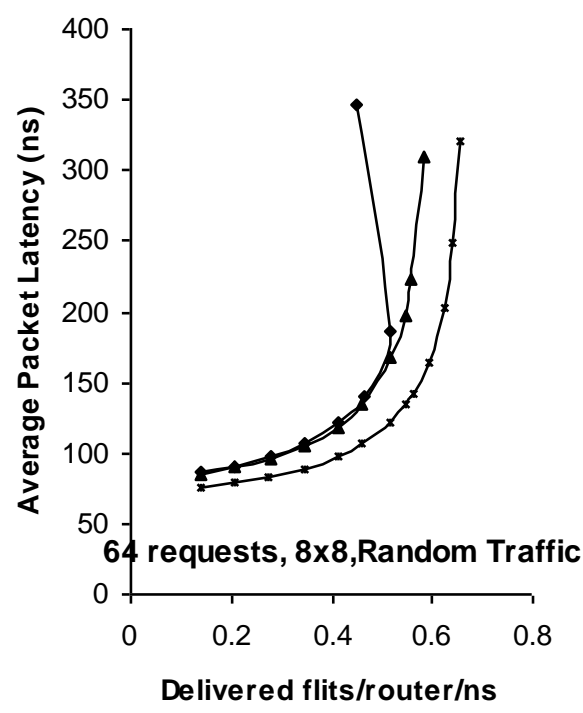

(b)

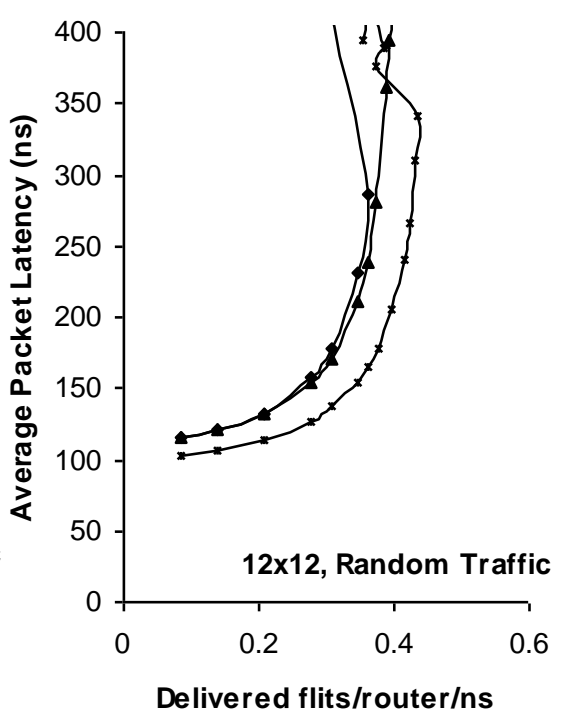

(c)

Figure 11. Scaling Results for the 21364 router.

Figure $11 \mathrm{~b}$ shows the results for the three arbitration algorithms for an $8 \times 8$ network with higher network load. Higher network load, in the form of greater number of outstanding misses, can be expected from future processors with deeper pipelines. Hence, this figure assumes 64 outstanding misses, four times higher than that of the 21364 processor. As the figure shows, even under such high network loads, SPAArotary outperforms both PIM1 and WFA-rotary. Thus, for example, at about roughly 200 nanoseconds of average packet latency, SPAA-rotary provides roughly $13 \%$ higher throughput compared to WFA-rotary.

Figure 11c shows the scaling results for the 21364 router for a 144-processor (12x12) network (Note: the 21364 network can only scale up to 128 processors). Like the first two scaling results, SPAA-rotary outperforms both PIM1 and WFA-rotary significantly. Thus, for a 200 nanoseconds average packet latency, SPAA-rotary provides an $18 \%$ higher throughput compared to WFA-rotary. Interestingly, however, at extremely high loads, SPAA-rotary is unable to prevent throughput degradation under saturation, whereas WFA-rotary's throughput continues to increase, possibly because of its synchronization between output port arbiters.

\section{CONCLUSIONS}

Large-scale cache-coherent shared-memory machines have become common server machines. Such machines often employ interconnection networks to allow communication between processors and memory modules. These interconnection networks must deliver low latency and high bandwidth to effectively run demanding parallel applications.

Interconnection networks usually consist of a fabric of interconnected routers, which receive packets arriving at their input ports and forward them to appropriate output ports. Unfortunately, network packets moving through these routers are often delayed due to conflicting demand for resources, such as output ports or buffer space. Hence, routers typically employ arbiters to resolve conflicting resource demands. These arbiters try to maximize the number of matches between packets waiting at input ports and free output ports.
Efficient design and implementation of these arbiters is critical to maximize network performance. The $1.2 \mathrm{GHz}$ implementation of the Alpha 21364 microprocessor's on-chip router, which can connect up to 128 processors in a $2 \mathrm{D}$ torus, made the already difficult task of designing arbitration algorithms even more challenging. Because the 21364's implementation allowed very few logic levels - between 12 and 13 per clock cycle - we had to carefully weigh the complexity of an arbitration algorithm against its benefit.

This paper proposed a new arbitration algorithm called SPAA (Simple Pipelined Arbitration Algorithm), which is implemented in the 21364 router's pipeline. Simulation results showed that SPAA significantly outperforms two earlier wellknown arbitration algorithms: PIM (Parallel Iterative Matching) and WFA (Wave-Front Arbiter), which is implemented in the SGI Spider switch. Instead of PIM, which is iterative and would have obviously performed poorly in the 21364 router, we considered PIM1, which runs only one iteration of the PIM algorithm.

SPAA outperformed PIM1 and WFA, even though both PIM1 and WFA have better matching capabilities than SPAA. This is because SPAA exhibits matching capabilities similar to PIM1 and WFA under realistic conditions when many output ports are busy, incurs fewer clock cycles to perform the arbitration, and can be pipelined effectively. We also demonstrated that SPAA will continue to deliver higher throughput compared to PIM1 and WFA, if the router were scaled to have twice the pipeline length, incur greater input load, or support bigger networks than the 21364 was designed for.

Additionally, we proposed a new prioritization policy called the Rotary Rule, which provided significant boost in network performance by preventing the network's adverse performance degradation from saturation at high network loads. The Rotary Rule prioritizes the movement of network packets already in the network over packets recently generated from the processor ports. We demonstrated the effectiveness of the Rotary Rule with WFA and SPAA. The Alpha 21364 router provides the Rotary Rule as an optional mode programmable at boot-time.

The arbitration algorithm choice for the Alpha 21364 router depends largely on its architectural constraints. The arbitration 
algorithm did not need to be as aggressive because of a maximum of two output port choices for each packet, perpacket arbitration, and virtual cut-through routing. Greater routing freedom, flit-level arbitration, and wormhole routing (with shallow buffering) may reduce the advantage of SPAA over PIM1 and WFA.

\section{Acknowledgements}

We thank Richard Kessler, who was one of the most important contributors to the design of the Alpha 21364 network. We also thank Geoff Lowney and the anonymous referees for their comments on earlier drafts of this paper. Finally, we thank the entire Asim team for their help with the Asim infrastructure.

\section{References}

[1] M.G.Ajmone Marshan, A. Bianco, and E. Leonardi, "RPA: A Flexible Scheduling Algorithm for Input Buffered Switches," IEEE Transaction on Communications, vol. 47, no. 12, pp. 1921-1933, Dec. 1999.

[2] Thomas E. Anderson, Susan S. Owicki, James B. Saxe, and Charles P. Thacker, "High Speed Switching for Local Area Networks," Fifth International Conference on Architectural Support for Programming Languages and Operating Systems, pp. 98 - 110, Boston, Massachusetts, October 12-15, 1992.

[3] Peter Bannon, "Alpha 21364: A Scalable Single-Chip SMP," $11^{\text {th }}$ Annual Microprocessor Forum, Microdesign Resources, Sebastopol, California, 1998.

[4] E.Baydal, P.Lopez, and J.Duato, "A Simple and Efficient Mechanism to Prevent Saturation in Wormhole Networks," $14^{\text {th }}$ International Parallel and Distributed Processing Symposium, pp. 617-622, 2000.

[5] Laxmi N. Bhuyan, "Analysis of Interconnection Networks with different arbiter designs," Journal of Parallel and Distributed Computing, vol. 4, no. 4, pp 384-403, August 1987.

[6] Alan Charlesworth, "The Sun Fireplane Interconnect," IEEE Micro, pp 36-45, Volume 22, Number 1, January/February 2002.

[7] Andrew Chien and Magda Konstantinidou, "Workloads and Performance Metrics for Evaluating Parallel Interconnects," IEEE TCCA Newsletter, Fall 1994.

[8] R.Cutler and S.Atkins, "IBM e-Server pSeries 680 Handbook," IBM, Armonk, N.Y., 2000; http://www.redbooks.ibm.com/pubs/pdfs/redbooks/sg246023.pdf.

[9] William J. Dally, "Virtual Channel Flow Control," 17 $7^{\text {th }}$ Annual International Symposium on Computer Architecture (ISCA), pp. 6068, 1990.

[10] William J. Dally and Charles L. Seitz, "The Torus Routing Chip," Distributed Computing, vol. 1, no. 4, pp. 187-196, Oct. 1986.

[11] A.DeHon, F. Chong, M. Becker, E. Egozy, H. Minsky, S. Peretz, and T.F.Knight Jr., "METRO: A Router Architecture for HighPerformance, Short-Haul Routing Networks," pp. 266-277, $21^{\text {st }}$ Annual International Symposium on Computer Architecture (ISCA), Chicago, Illinois, April 1994.

[12] H. Duan, J.W.Lockwood, S.M.Kang, and J.D.Will, "A High Performance OC12/OC48 Queue Design Prototype for Input Buffered ATM Switches," INFOCOM 97: $16^{\text {th }}$ Annual Joint Conference of the IEEE Computer and Communication Societies (Infocom 97), IEEE CS Press, pp. 20-28, Los Alamos, California, 1997.

[13] Jose Duato, "A New Theory of Deadlock-free Adaptive Routing in Wormhole Networks," IEEE Transaction on Parallel and Distributed Systems, vol. 4, no. 12, pp. 1320-1331, December 1993.

[14] Jose Duato, Sudhakar Yalamanchilli, and Lionel Ni, "Interconnection Networks: An Engineering Approach,” IEEE Computer Society, 1997.

[15] Joel Emer, Pritpal Ahuja, Nathan Binkert, Eric Borch, Roger Espasa, Toni Juan, Artur Klauser, Chi-Keung Luk, Srilatha Manne, Shubhendu S. Mukherjee, Harish Patil, and Steven Wallace, "Asim: A Performance Model Framework," IEEE Computer, pp. 68-76, February 2002.

[16] M. Galles, "Spider: A High-Speed Network Interconnect," IEEE Micro, vol. 17, no. 1, pp. 34-39, Jan.-Feb. 1997.

[17] K. Gharachorloo, et al., "Architecture and Design of the Alphaserver GS320," Architectural Support for Programming Languages and Operating Systems (ASPLOS), pp. 13-24, 2000.
[18] Paolo Giaccone, Devavrat Shah, and Balaji Prabhakar, "An Implementable Parallel Scheduler for Input-Queued Switches," IEEE Micro, pp 19-25, Volume 22, Number 1, January/February 2002.

[19] Hewlett-Packard, "Meet the HP Superdome Servers," September, 2001; http://www.hp.com/products1/servers/scalableservers/superdome/infolibrary/whitepapers/technical_wp.pdf.

[20] M.D.Hill, "A Case for Direct-Mapped Caches," IEEE Computer, vol. 21, no. 12 , pp. 25-40, December 1988.

[21] P.Lopez, J.M.Martinez, J.Duato, "DRIL: Dynamically Reduced Message Injection Limitation Mechanism for Wormhole Networks," International Conference on Parallel Processing, pp. 535-542, August 1998.

[22] P.Lopez, J.M.Martinez, J.Duato, and F.Petrini, "On the Reduction of Deadlock Frequency by Limiting Message Injection in Wormhole Networks," Parallel Computer Routing and Communication Workshop, June 1997.

[23] N. McKeown, "iSLIP: A Scheduling Algorithm for Input-Queued Switches," IEEE Transaction on Networking, vol. 7, no. 2, pp. 188201, April 1999.

[24] N. McKeown, "Scheduling Algorithms for Input-Queued Cell Switches," doctoral dissertation, Dept. of EECS, University of California, Berkeley, 1995.

[25] Devavrat Shah, Paolo Giaccone, and Balaji Prabhakar, "Efficient Randomized Algorithms for Input-Queued Switch Scheduling," IEEE Micro, pp 10-18, Volume 22, Number 1, January/February 2002.

[26] Shubhendu S. Mukherjee, Peter Bannon, Steven Lang, Aaron Spink, and David Webb, "The 21364 Network Architecture," IEEE Micro, pp 26-35, Volume 22, Number 1, January/February 2002.

[27] Li-Shiuan Peh and William J. Dally, "A Delay Model for Speculative Architecture for Pipelined Routers," $7^{\text {th }}$ Annual International Symposium on High-Performance Computer Architecture (HPCA), pp. 255266, Neuvo Leon, Mexico, January 2001.

[28] G.E.Pfister and V.A.Norton, "Hot-Spot Contention and Combining in Multistage Interconnection Networks," IEEE Transaction on Computers, C-34(10):943-948, October 1985

[29] Parthasarathy Ranganathan, Kourosh Gharachorloo, Sarita V. Adve, and Luiz Andre Barroso, "Performance of Database Workloads on Shared-Memory Systems with Out-of-Order Processors," Eighth International Conference on Architectural Support for Programming Language (ASPLOS), pp. 307-318, San Jose, California, October 1998.

[30] S.L.Scott and G. Sohi, "The Use of Feedback in Multiprocessors and its Application to Tree Saturation Control," IEEE Transactions on Parallel and Distributed Systems, vol. 1, no. 4, pp. 385-398, October 1990.

[31] S.L.Scott and G.M.Thorson, "The Cray T3E Network," Hot Interconnects IV, pp. 147-156, 1996.

[32] Simon Steely, Compaq Computer Corporation, Personal Communication.

[33] Silicon Graphics, "SGI 3000 Family Reference Guide," 2001; http://www.sgi.com/origin/3000/3000_ref.pdf.

[34] A. Smai and L.Thorelli, "Global Reactive Congestion Control in Multicomputer Networks," $5^{\text {th }}$ International Conference on High Performance Computing, pp. 179-186, 1998.

[35] C. B. Stunkel, J. Herring, B. Abali, and R. Sivaram, “'A new switch chip for IBM RS/6000 SP systems," Supercomputing '99, Portland, Oregon, November 1999.

[36] Yuval Tamir and Hsin-Chou Chi, "Symmetric Crossbar Arbiters for VLSI Communication Switches," IEEE Trans. on Parallel and Distributed Systems, vol. 4, no. 1, pp. 13-27, January 1993.

[37] J.M.Tendler, et al., "IBM e-server POWER4 System Microarchitecture," IBM, Armonk, N.Y., 2001; http://www1.ibm.com/servers/eserver/pseries/hardware/whitepapers/power4.pdf.

[38] Mithuna Thottethodi, Alvin Lebeck, and Shubhendu S. Mukherjee, "Self-Tuned Congestion Control for Multiprocessor Networks," Seventh Annual International Symposium on High-Performance Computer Architecture (HPCA), pp. 107-118, Monterrey, Mexico, January 2001

[39] Brian Towles and William J. Dally, "Worst-case Traffic for Oblivious Routing," Computer Architecture Letters, (http://www.cs.virginia.edu/ tcca/2002paps.html), 2002. 